\title{
ESTUDO DA TRANSFERÊNCIA DE MASSA DE UM PRATO PERFURADO INDUSTRIAL NA DESTILAÇÃO DO ETANOL ATRAVÉS DE CFD
}

\author{
G. C.OLIVEIRA ${ }^{1}$, G. H.JUSTI ${ }^{1}$, G. C.LOPES ${ }^{1}$ e J. A. S. GONÇALVES ${ }^{1}$ \\ ${ }^{1}$ Universidade Federal de São Carlos, Departamento de Engenharia Química \\ E-mail para contato: gabrielhjusti@yahoo.com.br
}

\begin{abstract}
RESUMO - A destilação é uma das mais importantes técnicas de separação de componentes empregada a nível industrial nos mais diversos processos. Estudos empíricos em colunas de destilação são raros, em parte devido às grandes dimensões destes aparelhos e o seu alto investimento. Neste estudo, foi proposta a simulação computacional de pratos perfurados de destilação usando ferramentas de CFD, tendo como principal objetivo conceber um modelo capaz de prever a hidrodinâmica do prato e a transferência do componente mais volátil entre o líquido e o vapor. Para isso, foram usados modelos 3D, transientes e multifásicos com abordagem Euler-Euler. Os principais resultados mostram a fração mássica e eficiência do estágio, os quais apresentaram concordância quando comparados com a literatura, obtendo-se uma eficiência de estágio de $65 \%$. CFD demonstrou ser uma ferramenta valiosa para estudar o fluxo em internos de torres de destilação.
\end{abstract}

\section{INTRODUÇÃO}

A destilação é uma das mais antigas operações unitárias de separação física de uma mistura. Além de ser bastante empregada na produção de etanol, esta técnica também é muito utilizada na indústria de processos químicos, abrangendo cerca de 90 a 95\% das separações (Wankat, 2007). A principal forma de avaliar a qualidade de um processo de destilação dá-se através da capacidade de extrair o componente mais volátil da fase rica. (Kister, 1992).

Com isso, muitos autores utilizaram as técnicas da Fluidodinâmica Computacional (CFD) para verificar a transferência de massa em pratos perfurados de coluna de destilação. Neste contexto, podese citar os principais trabalhos como de Rahimiet al. (2006), Noriler et al.(2010) e Rahimi et al. (2012), os quais predisseram a eficiência de separação no estágio de destilação e os campos de fração mássica dos componentes distribuídos ao longo do prato perfurado.

\section{MODELAGEM MATEMÁTICA}

Neste estudo, um modelo de CFD multifásico e multicomponente foi utilizado para descrever a hidrodinâmica e a transferência das espécies químicas entre as fases para um sistema etanol-água a 1 atm utilizando uma abordagem Euleriano-Euleriano. As equações que descrevem o fenômeno são 
apresentadas abaixo, onde os subscritos $\alpha$ e $\beta$ representam as fases líquida e vapor:

\subsection{Equação da Continuidade}

$$
\frac{\partial}{\partial t}\left(f_{\alpha} \rho_{\alpha}\right)+\nabla \cdot\left(f_{\alpha} \rho_{\alpha} \mathbf{v}_{\alpha}\right)=\boldsymbol{S}_{M S, \alpha}+\sum_{\beta=1}^{N_{\mathrm{f}}} \Gamma_{\alpha \beta}
$$

onde $f, \rho$ e $\mathbf{v}$ representam a fração volumétrica, massa específica e o vetor velocidade para a fase $\alpha$, respectivamente. $S_{M S}$ representa o termo de fonte de massa, como não há reações ou outras formas de criação de massa para este escoamento, este termo é nulo. O termo $\Gamma$ representa a taxa de transferência de massa entre as fases por unidade de volume.

\subsection{Equação da Quantidade de Movimento}

$$
\begin{aligned}
\frac{\partial}{\partial t}\left(f_{\alpha} \rho_{\alpha} \mathbf{v}_{\alpha}\right)+ & \nabla \cdot\left[f_{\alpha}\left(\rho_{\alpha} \mathbf{v}_{\alpha} \otimes \mathbf{v}_{\alpha}\right)\right] \\
& =-f_{\alpha} \nabla p_{\alpha}+\nabla \cdot\left(f_{\alpha} \mathbf{T}_{\alpha}^{\mathrm{eff}}\right)+\sum_{\beta=1}^{N_{\mathrm{f}}}\left(\Gamma_{\alpha \beta}^{+} \mathbf{v}_{\beta}-\Gamma_{\beta \alpha}^{+} \mathbf{v}_{\alpha}\right)+\boldsymbol{S}_{M, \alpha}+\boldsymbol{M}_{\alpha}
\end{aligned}
$$

onde $p$ representa a pressão, na qualtodas as fases compartilham o mesmo campo de pressão, isto é, $p_{\alpha}=p_{\beta}=p . \mathbf{T}^{\mathrm{eff}}$ representa o tensor efetivo e $\left(\Gamma_{\alpha \beta}^{+} \mathbf{v}_{\beta}-\Gamma_{\beta \alpha}^{+} \mathbf{v}_{\alpha}\right)$ representa a transferência de momento entre as fases devido à transferência de massa entre elas.O termo $\boldsymbol{S}_{M}$ representa a fonte de Momentum, em virtude das forças externas do corpo e/ou fontes definidas e o termo $\boldsymbol{M}_{\alpha}$ descreve as forças interfaciais atuando nas fases devido à presença de outra fase.

\subsection{Equação das Espécies Químicas}

$$
\begin{aligned}
\frac{\partial}{\partial t}\left(f_{\alpha} \rho_{\alpha} y_{A, \alpha}\right) & +\nabla \cdot\left\{f_{\alpha}\left[\rho_{\alpha} \mathbf{v}_{\alpha} y_{A, \alpha}-\rho_{\alpha} D_{A, \alpha}\left(\nabla y_{A, \alpha}\right)\right]\right\} \\
& =S_{A, \alpha}+\sum_{\alpha \neq \beta}^{N_{\mathrm{f}}} \boldsymbol{Y}_{A, \alpha \beta}+\sum_{\alpha \neq \beta}^{N_{\mathrm{f}}}\left(\Gamma_{\alpha \beta}^{+} y_{A, \beta}-\Gamma_{\beta \alpha}^{+} y_{A, \alpha}\right)
\end{aligned}
$$

onde $y_{A, \alpha}$ e $D_{A, \alpha}$ representam a fração mássica e a difusividade do componente $\mathrm{A}$ na fase $\alpha$, respectivamente.Os termos $\boldsymbol{Y}_{A, \alpha \beta}$ e $\left(\Gamma_{\alpha \beta}^{+} y_{A, \beta}-\Gamma_{\beta \alpha}^{+} y_{A, \alpha}\right)$ representam a transferência das espécies químicas entre as fases, o primeiro devido à diferença de potencial químico e o segundo devido à quantidade líquida de massa total transferida de uma fasepara a outra. 


\subsection{Equações de Restrição}

As frações mássicas dos componentes e as frações volumétricas das fases podem ser relacionadas através de uma restrição de soma, na qual a somatória das frações deve ser igual 1.

\subsection{Hipóteses do modelo}

As seguintes hipóteses foram consideradas para a modelagem do prato perfurado: um sistema de duas fases; a fase gasosa é a fase dispersa; a mistura binária etanol-água foi utilizada; a taxa líquida de transferência de massa entre as fases é tão pequena que pode ser desprezada. Neste caso, o termo do lado direito da Equação 1 desaparece e os termos associados a ele nasEquações 2 e 3 são nulos; e para a fase dispersa não foi utilizado nenhum modelo de turbulência.

\subsection{Equações de Fechamento}

Transferência de quantidade de movimento na interface:Em escoamento líquido-vapor em pratosperfurados de destilação, a força de arraste destaca-se por possuir maior importância nos fenômenos de transferência de momento na interface. Portanto, para modelar o coeficiente de arraste, foi utilizado a correlação de Krishna et al. (1999), na qual torna-se o termo de transferência de quantidade de movimento na interface independente do diâmetro de bolha.

Transferência das espécies químicas na interface: $\mathrm{O}$ modelo de duas resistências foi utilizado para determinar o $\boldsymbol{Y}_{A, \alpha \beta}$, conforme apresentado pela Equação 5 .

$$
\boldsymbol{Y}_{A, \alpha \beta}=-\boldsymbol{Y}_{A, \beta \alpha}=k_{\alpha \beta}^{C} A_{\alpha \beta}\left(K^{C} \rho_{A, \alpha}-\rho_{A, \beta}\right) \quad e \quad \frac{1}{k_{\alpha \beta}^{C}}=\frac{1}{k_{\alpha}^{C}}+\frac{K^{C}}{k_{\beta}^{C}}
$$

onde $K^{C}$ é a constante de equilíbrio termodinâmico e $k_{\alpha}^{C}$ e $k_{\beta}^{C}$ representam os coeficientes de transferência de massa.

A teoria de penetração de Higbie (1953) foi utilizada para calcular os coeficientes de transferência de massa e a constante de equilíbrio termodinâmico pode ser obtida da literatura.

Modelo de turbulência: O modelo Shear Stress Transport (SST) foi utilizado para a fase contínua, pois trata-se de um modelo que engloba o modelo $k-\varepsilon$ padrão e o modelo $k-\omega$. Sua vantagem é a flexibilidade e o tratamento dado próximo às paredes, além de apresentar melhor convergência.

\section{METODOLOGIA}

\subsection{Domínio Computacional e Malha Numérica}

O domínio computacional desenvolvido foi um prato perfurado de coluna de 
destilaçãoutilizando o software DesignModeler (ANSYS 14.5), suas características geométricas foram baseadas no trabalho de Solari e Bell (1986). O prato perfurado possui um diâmetro de 1,213 [m] e espaçamento de 0,61 [m], vertedor com altura de 0,05 [m] e a entrada de líquido com altura de 0,038 [m]. O diâmetro dos orifícios é de 0,0127 [m] e foramdistribuídos de forma triangular com 0,05 [m] de distância entre eles, totalizando 348 orifícios.

Na construção da malha foi utilizado o softwareMeshing (ANSYS 14.5) e foi realizadoteste de sensibilidade da solução da malha, visando garantir independência dos resultados em relação ao seurefinamento. Trata-se de uma malha mista/híbrida, ou seja, com tetraédros (região dos orifícios) e hexaédros. O refino da malha deu-se na região de borbulhamento eforam realizados 4 tipos de refino, variando de 600.000 à 1.800.000 elementos. Para avaliar a sensibilidade da malha foi escolhido o parâmetro hidráulico altura de líquido claro. A partir de 1.200.000 elementos, a variação da altura de líquido claro é pequena, na ordem $0,05 \mathrm{~mm}$, sugerindo que maior refinamento não se faz necessário.A Figura 1 ilustra o domínio computacional e a malha numérica utilizada.

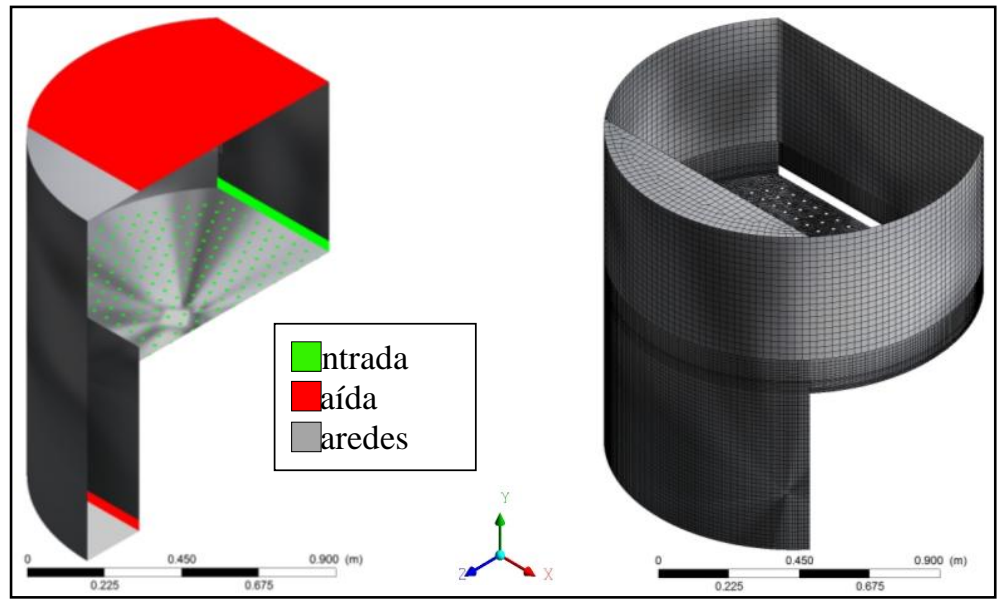

Figura 1 - Prato perfurado de destilação: condições de contorno (esq.) e malha (dir.).

Com base nesta análise, a malha com 1.200 .000 elementos foi empregada para obter os resultados.

\subsection{Condições de Inicialização e de Contorno}

As condições de contorno e iniciais são fundamentais para o fechamento domodelo e permitir a resolução das equações numéricas envolvidas nas simulações. A Figura 1 ilustra as regiões de contorno do prato perfurado.

Inicialização do domínio: A condição inicial aplicada na simulação consistiu em velocidades nulas, ou seja, com o domínio vazio, permitindo a observação do fenômeno de enchimento do prato.

Entrada de vapor: Considerou-se que a vazão mássica de vapor que entra no prato pelos orifícios é a mesma em cada orifício. Sendo assim, a velocidade de vapor fluindo através dos 
furos $\left(\mathrm{v}_{y, V}^{\text {in }}\right)$ para a região “Entrada de Vapor” foi calculada conforme a Equação 6:

$$
\mathrm{v}_{y, V}^{i n}=\frac{U_{S} A_{B}}{N_{H} A_{H, i}}
$$

onde $U_{S}, A_{B}, N_{H}$ e $A_{H, i}$ representam a velocidade superficial do gás, área de borbulhamento, número total de orifícios e área de um orifício, respectivamente. Considerou-se que apenas vapor entra por esta região, pois a quantidade arrastada de líquido pelo vapor é desprezível. Adicionalmente, para o sistema binário etanol-água, especificou-se a fraçãomássica de etanolna entrada sendo igual a 0,812.

Entrada de líquido: Adotou-se um perfil de velocidade uniforme para o líquido ( $\mathrm{v}_{z, L}^{\text {in }}$ )para aregião "Entrada de Líquido" do prato, conforme a Equação 7:

$$
\mathrm{v}_{z, L}^{i n}=\frac{Q_{L}}{\mathrm{~A}_{L}^{i n}}
$$

onde $Q_{L}$ e $A_{L}^{\text {in }}$ representam a vazão volumétrica de líquido e área de entrada de líquido no prato, respectivamente. Considerou-se que apenas líquido entra no domínio por esta região, pois a quantidade arrastada de vapor é desprezível. Adicionalmente para o binário etanol-água, especificouse a fraçãomássica de etanolna entrada sendo igual a 0,785 .

Saída de gás: Adotou-se uma condição de pressão e especificou-se uma pressão absoluta de 1 $\operatorname{atm}\left(p_{\text {relativa }}=0 \mathrm{~atm}\right)$ para aregião "Saída de Vapor" do prato.

Saída de líquido: Adotou-se também uma condição de pressão para aregião "Saída de Líquido" do prato. A ideia foi adicionar uma resistência na saída de líquido que existe em função do prato consequente, o qual não consta no modelo proposto e foi projetada para representar a coluna de líquido que forma na região do downcomer doprato com ape aproximadamente $20 \mathrm{~cm}$ de altura.

Paredes: A condição de não deslizamento para a região "Paredes" para as duas fases.

A análise de CFD foi realizada no software CFX-Pre 14.5. A condição operacional utilizada para o líquido e vapor na simulação foi $Q_{L}=6,94 \times 10^{-3}\left[\mathrm{~m}^{3} \mathrm{~s}^{-1}\right]$ e $F_{S}=1,015\left[\mathrm{~m} \mathrm{~s}^{-1}\left(\mathrm{~kg} \mathrm{~m}^{-3}\right)^{1 / 2}\right]$, respectivamente. $\mathrm{O}$ fator $F_{S}$ é função da velocidade superficial do vapor e da massa específica do vapor, portanto é definida como $F_{s}=U_{s} \sqrt{\rho_{\mathrm{v}}}$. A simulação foi realizada em um AMD Opteron $^{(\mathrm{TM})} 2,26 \mathrm{GHz}(25 \times 2,26 \mathrm{GHz})$,em paralelo, para simular40 segundos do escoamento do prato perfurado.

\section{RESULTADOS}

\subsection{Campos e Perfis de Frações Mássicas}


Visando conhecer a distribuição da concentração de etanol no líquido por todo o prato e ao longo do tempo simulado, a Figura 2 ilustra a distribuição de concentrações para instantes de tempo definidos.

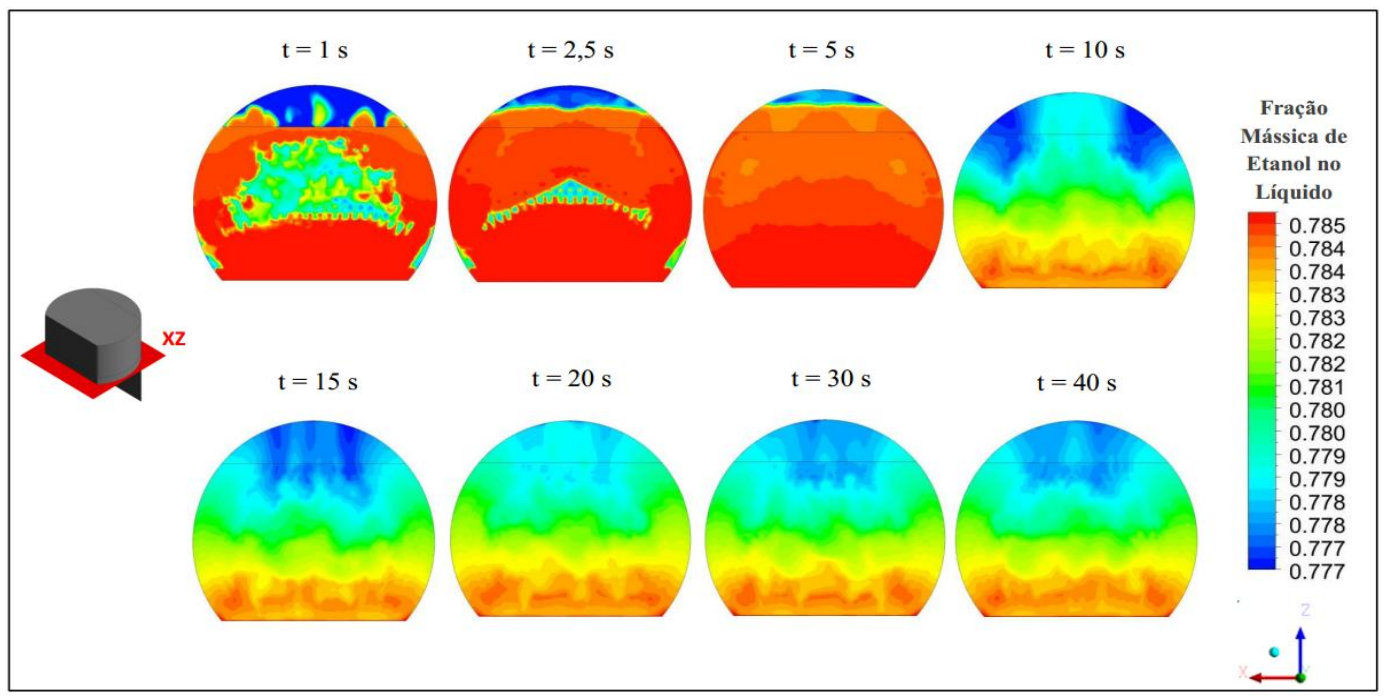

Figura 2 - Distribuiçãodos campos de fração mássica de etanol no líquido ao longo do tempo em um plano ZX $(\mathrm{y}=0,038 \mathrm{~m})$.

Nos primeiros instantes, o perfil ainda não está definido, pois o prato está no seu período puramente transiente. Decorridos cerca de $10 \mathrm{~s}$, um visível perfil começa a ser moldado, evidenciando regiões com concentrações distintas. O líquido entra no prato com fração mássica alta (regiões em vermelho) e se "empobrece" em virtude da troca de espécies entre fases. Tem-se que a diminuição do componente na fase líquida dá-se de forma gradual ao longo do prato, uma vez que o processo de transferência é função do tempo de contato, área efetiva de contato e gradiente de concentração. Estima-se uma redução mais expressiva na região central do prato, enquanto as regiões periféricas demonstram uma troca levemente mais lenta. Acredita-se que as trocas na região periférica do prato sejam influenciadas pela existência dos efeitos de recirculação, estagnação, baixa mistura ou o próprio efeito de parede.

A Figura 3 apresenta a variação temporal da fração mássica média de etanol para as fases líquida e vapor, avaliadas nas saídas do domínio computacional.Observando-se o comportamento da fração mássica de etanol em ambas as fases, nota-se variações diferentes ao longo do tempo. A fase gasosa (entra no prato com 0,812) varia quase que linearmente crescente até atingir um patamar oscilatório depois de decorridos aproximadamente 10s. Após este tempo, a variável oscila em torno de um valor médio de 0,861 ilustrando o regime chamado de quase estacionário. Através de uma média representativa da fração mássica de etanol, estima-se o enriquecimento da fase gasosa em aproximadamente $6 \%$. Em relação à fase líquida, a variação da fração mássica de etanol é mais abrupta nos primeiros 6 s de escoamento real, passando de 0,712 para 0,777. Este comportamento deve-se ao fato de o monitoramento ter sido iniciado com o prato vazio. De qualquer forma, após o estabelecimento do fluxo no prato, a fração de etanol no líquido permanece quase que inalterada em 
aproximadamente 0,778 . Ou seja, o líquido estabiliza-se com um valor pouco menor ao de entrada (redução de aproximadamente 1\%), indicando a perda do componente mais volátil para a fase gasosa.

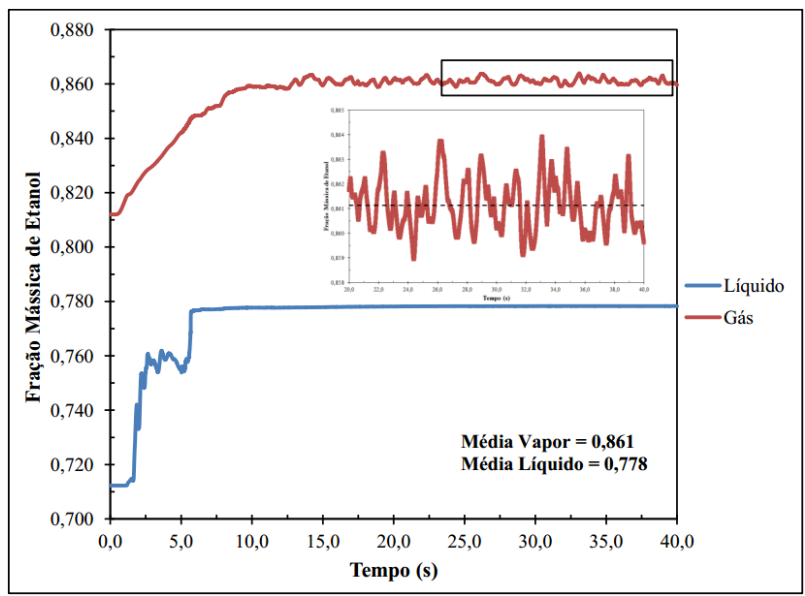

Figura 3-Variação da fração mássica de etanol para as fases líquida e vapor ao longo do tempo.

Como análise da eficiência de separação no estágio simulado, a Figura 4apresenta o comportamento médio da Eficiência de Murphree ao longo do tempo.
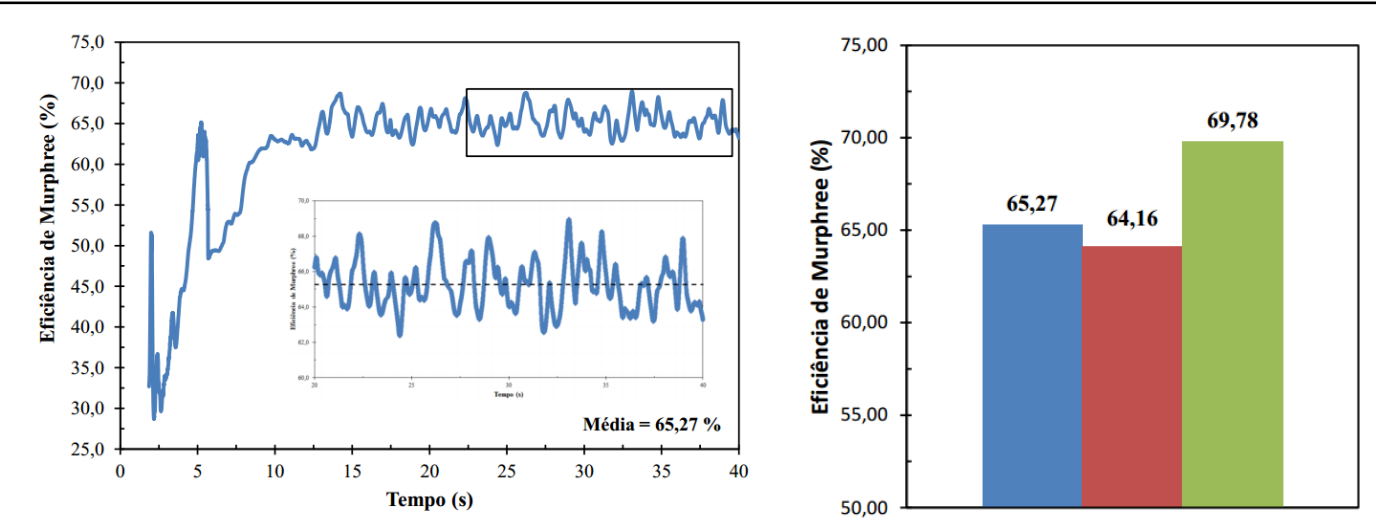

$\square$ CFD

Noriler (2010)

van Winkle, MacFarland e Sigmund (1972)

Figura 4 - Variação temporal da eficiência de Murphree no prato (esq.) e a comporação entre as eficiência simulada com a literatura e correlações empirícas.

Os dados aqui encontrados estão de acordo com aqueles citados por Norileret al. (2010), que apresentou uma diferença de $1,7 \%$ de desvio. Acredita-se que esta discrepância dos resultados obtidos numericamente dê-se por dois motivos: primeiro e principalmente pelo tamanho e características do prato avaliado e segundo pelas difusividades estipuladas. $\mathrm{O}$ menor diâmetro do prato avaliado por Noriler et al.(2010) e um vertedor mais alto, provavelmente permitem um tempo menor de contato entre as fases e aumenta as chances de recirculação. Somados a isso, as difusividades estipuladas diferiram-se nos valores, o que provavelmente acarretou taxas diferentes de 
transferência de espécies químicas. Por outro lado, a correlação de van Winkle et al.(1972) fornece eficiências superiores ao modelo numérico aqui proposto, com 6,5\% de desvio.

Acredita-se que este desvio é aceitável, pois a correlação utilizada é sensível aos valores das propriedades da mistura líquida.

\section{CONCLUSÕES}

A metodologia proposta neste trabalho mostrou ser adequada para descrever a eficiência de separação de um escoamento líquido-vapor em um prato perfurado de coluna de destilação. Foi possível calcular a eficiência estágio baseado em modelos que consideram as variações espaciais e temporais ao longo do domínio computacional. Isso é importante em termos de projeto e otimização de pratos perfurados de destilação.Finalmente, as técnicas de CFD podem ser utilizadas para otimizar projetos de pratos e suas condições operacionais desses processos.

\section{AGRADECIMENTOS}

Os autores agredecem a CNPq e a CAPES pelo suporte financeiro.

\section{REFERÊNCIAS}

HIGBIE, R. The rate of absorption of a pure gas into a still liquid during short periods of exposure. Transactions AIChE, v. 31, p. 365-389, 1935.

KISTER, H. Z. Distillation design. Boston: McGraw-Hill, 1992.

KRISHNA, R.; URSEANU, M. I.; VAN BATEN, J. M.; ELLENBERGER, J. Rise velocity of a swarm of large gas bubbles in liquids. Chem. Eng. Science, v. 54, p. 171-183, 1999.

NORILER, D; BARROS, A. A. C.; MACIEL, M. R. W.; MEIER, H. F.Simultaneous momentum, mass, and energy transfer analysis of a distillation sieve trayusing CFD techniques: prediction of efficiencies. Industrial and Engineering Chemistry Research, v. 49, p. 6599-6611, 2010.

RAHIMI, R.; RAHIMI, M. R.; SHAHRAKI, F.; ZIVDAR, M. Efficiencies of sieve tray distillation columns by CFD simulation. Chem. Eng. Technol., 29, n. 3, p. 326-335, 2006.

RAHIMI, R.; SOTOODEH, M. M.; BAHRAMIFAR, E. The effect of tray geometry on the sieve tray efficiency. Chemical Engineering Science, v. 76, p. 90-98, 2012.

SOLARI, R. B.; BELL, R. L. Fluid flow patterns and velocity distribution on commercialscale sieve trays. AIChE Journal, v. 32, n. 4, p. 640-649, 1986.

VAN WINKLE, M.; MACFARLAND, S. A.; SIGMUND, P. M. Predict distillation efficiency.Hydrocarbon Processing, v. 51, n. 7, p. 111-114, 1972. 
19 a 22 de outubro de 2014

Florianópolis/SC

WANKAT, P. C. Separation process engineering. 2 ed. Upper Saddle River: Prentice Hall, 2007. 